\title{
Field Prototype of the ENEA Neutron Active Interrogation Device for the Detection of Dirty Bombs
}

\author{
Nadia Cherubini ${ }^{1, *}$, Alessandro Dodaro ${ }^{1}$, Giada Gandolfo ${ }^{2}$, Luigi Lepore ${ }^{2}$, \\ Giuseppe A. Marzo ${ }^{1}$, Ermanno Piccinelli ${ }^{1}$ and Romolo Remetti ${ }^{2}$ \\ 1 ENEA, Italian National Agency for New Technologies, Energy and Sustainable Economic Development, \\ C.R. Casaccia, via Anguillarese 301, Roma 00123, Italy; alessandro.dodaro@enea.it (A.D.); \\ giuseppe.marzo@enea.it (G.A.M.); ermanno.piccinelli@enea.it (E.P.) \\ 2 Department of Basic and Applied Sciences for Engineering, SAPIENZA University of Roma, via Scarpa 14, \\ Roma 00161, Italy; giada.gandolfo@uniroma1.it (G.G.); luigi.lepore@uniroma1.it (L.L.); \\ romolo.remetti@uniroma1.it (R.R.) \\ * Correspondence: nadia.cherubini@enea.it; Tel.: +39-06-3048-6662
}

Academic Editor: Palmiro Poltronieri

Received: 14 June 2016; Accepted: 14 October 2016; Published: 19 October 2016

\begin{abstract}
The Italian National Agency for New Technologies, Energy, and Sustainable Economic Development (ENEA) Neutron Active Interrogation (NAI) device is a tool designed to improve CBRNE defense. It is designed to uncover radioactive and nuclear threats including those in the form of Improvised Explosive Devices (IEDs), the so-called "dirty bombs". The NAI device, at its current development stage, allows to detect $6 \mathrm{~g}$ of ${ }^{235} \mathrm{U}$ hidden in a package. It is easily transportable, light in weight, and with a real-time response. Its working principle is based on two stages: (1) an "active" stage in which neutrons are emitted by a neutron generator to interact with the item under inspection, and (2) a "passive" stage in which secondary neutrons are detected originating a signal that, once processed, allows recognition of the offence. In particular, a clear indication of the potential threat is obtained by a dedicated software based on the Differential Die-Away Time Analysis method.
\end{abstract}

Keywords: neutrons; neutron interrogation; neutron generator; special nuclear materials; security; improvised explosive device; dirty bomb; fissile; fertile

\section{Introduction}

Nowadays, the current threat of international terrorism is set to a severe level, demanding worldwide enhanced security. As a direct consequence, the scientific community is strongly required for both preventive foiling of illicit traffic of dangerous substances, and managing hazardous events.

A major concern is about attacks to people in crowded places by means of explosives, radioactive, or bio-hazardous weapons. This led to the blooming of research programs oriented in conceiving and prototyping devices able to achieve early detection of such kind of offences. This research field is relatively young, and technical solutions seem to be currently at a first-of-a-kind level. In the last few years, detection of Special Nuclear Materials (SNMs) in freight has been one of the most critical security issues for the European countries. In this context, the European Commission 7th Framework Research Programme on Secure Societies funded the EDEN (End-user driven DEmo for cbrNe) Demonstration Project, whose primary objective is to provide solutions for improving CBRNE (chemical, biological, radiological, nuclear or explosives) resilience capacity of the EU society.

Since September 2013, ENEA—-the Italian National Agency for New Technologies, Energy, and Sustainable Economic Development-has been involved in the EDEN project in order to develop advanced and innovative tools for the detection of explosives, the prevention of threats to nuclear plants, the simulation of the dispersion of contaminants in the atmosphere, and the management of 
emergency situations. In particular, the Nuclear Material Characterization and Radioactive Waste Management Laboratory has been involved in the design and realization of the Neutron Active Interrogation (NAI) sensor whose purpose is the non-destructive inspection of samples suspected of containing fissile or fertile material and explosives. An example of the application of such an instrument is the illicit trafficking of SNMs or the detection of Improvised Explosive Devices (IEDs), e.g., made of natural uranium combined with explosives potentially designed for spreading radioactive contamination to harm the population.

In this paper we focus on the design of NAI for the detection of fissile material. NAI working principle is based on two stages: (1) an active stage in which neutrons are emitted by a neutron generator to interact with the target under inspection, and (2) a passive stage in which secondary neutrons are detected. Appropriate developed software analyzes the measured signals and visualizes the results in a user-friendly manner, giving the operator a clear indication of the potential threat. The system is intended as a transportable device. It is relatively light in weight, loadable on remote handlers, and versatile for a large range of suspect items. This paper describes the NAI at the current stage of development and its performances. The performance analysis has been carried out using a Monte Carlo code for radiation transport. Such simulation results have been used during the device optimization process allowing the authors to propose an experimental setup that has been tested on the field.

\section{The Detection of Explosives and SNMs}

To date, the detection of explosive devices has been entrusted to the identification of chemical components typical for the detonating stage, or the identification of electronics and wiring specific to this kind of devices.

Alongside the employ of bags and containers, radiography, or sniffer dogs, nowadays nuclear technologies can strongly contribute to the identification of explosives. They are based on: (1) backscattering techniques [1]; (2) fast neutron spectroscopy [2]; (3) Thermal Neutron Analysis (TNA) [3]; (4) Fast Neutron Analysis (FNA) [4]; and (5) Associated Particle Imaging (API) [5].

Runkle et al. [6] reviewed a variety of detection systems for chemical explosives oriented for air-cargos detection, highlighting the current paucity of solutions and the necessity of major improvements in this research field. Currently, the main recent solutions are: (1) photon-based interrogation analysis; (2) neutron-based interrogation analysis; and (3) laser-based optical interrogation analysis. Starting from the latter, Hakonen et al. [7] reviewed the Raman spectrometry performances showing its capabilities for real-time identification of illicit substances on contaminated surfaces. Photon-bases interrogation techniques deal with radiographic analysis of the sample (e.g., check-in luggage screening at the airport), and photon-induced analysis (e.g., X-ray diffraction, [8]). Regarding devices based on the neutron interrogation techniques for chemicals and explosives identification, a few devices have been developed, mostly for specialized use in the military field [9-12].

The NAI passive stage for detection of fissile materials is based on the Differential Die-Away Time Analysis (DDAA hereafter). DDAA working principle and its feasibility have been demonstrated by [13]; however, such a technique has usually been limited to the detection of plutonium and uranium fissile isotopes in samples or waste drums in controlled environments, such as characterization laboratories, waste management sites, fuel reprocessing plants. As an alternative to DDAA, other different techniques could have potentially been adopted for detecting plutonium and uranium fertile isotopes (e.g., Neutron Coincidence Counting, Neutron Multiplicity Counting), but they all imply longer measurements for obtaining statistically significant data [14]. Jordan et al. [15-18] experimentally proved the ability of the fast neutron interrogation technique and the DDAA for a prompt detection of fissile material. Experiments were carried out providing evidence that the approach allows the detection of hundreds of grams of ${ }^{235} \mathrm{U}$ in laboratory setups although they are heavy in weight, with large masses of moderator and structural materials. Currently, no commercial device which is based on such an approach is available. Commercial devices using different techniques 
are based on the observation of spontaneous, or neutron-induced, photon emission, preventing the detection of SNMs in real time.

Analyzing performances and limitation of the state-of-the-art devices available on the market, there is evidence that a fast, reliable, yet accurate detection of IEDs targeted for nuclear material dispersion, appears to be easily provided by the detection of fissile material, instead of chemical or explosive identification. Regarding the detection of fissile material, any field device existing today, and the ENEA NAI device herein proposed, could be considered as a first-of-a-kind based on their current development level.

\section{The ENEA Neutron Active Interrogation device}

The ENEA NAI device is the combination of a neutron source, a moderator around the sample suspected of containing fissile material, and a neutron detector array. The advantages of using a neutron generator as neutron source over a radioisotope source are its large yields and the source modulation flexibility. Moreover, the generator can be switched off simplifying transportation and operations.

The neutron generator consists of a compact linear accelerator that accelerates deuterium (d) ions toward a target which contains tritium ( $\mathrm{t}$ ); the $\mathrm{d}-\mathrm{t}$ fusion reaction generates neutrons of $14.1 \mathrm{MeV}$ (i.e., fast neutrons).

The detector system is an array of ${ }^{3} \mathrm{He}$ tube proportional counters based on the ${ }^{3} \mathrm{He}(\mathrm{n}, \mathrm{p})^{3} \mathrm{H}$ reaction. Since neutrons are not ionizing particles, their detection depends on the ionizing properties of the charged particles they produce in the nuclear reaction. ${ }^{3} \mathrm{He}$ tubes are the most common because the reaction ${ }^{3} \mathrm{He}(\mathrm{n}, \mathrm{p})^{3} \mathrm{H}$ has the highest cross section among the possible reactions used for neutron detection $\left({ }^{10} \mathrm{~B}(\mathrm{n}, \alpha),{ }^{6} \mathrm{Li}(\mathrm{n}, \alpha),{ }^{3} \mathrm{He}(\mathrm{n}, \mathrm{p})\right)$. Since the cross section is higher for thermal neutron energies, the ${ }^{3} \mathrm{He}$ tubes are generally enclosed into moderator blocks (e.g., polyethylene blocks), to increase the detection efficiency for higher neutron energies.

The interrogation technique can be summarized as follows: (1) the neutron generator emitting an appropriate flux of fast neutrons, (2) neutrons slowing down in the moderator occurring along the trajectory to the sample to be analyzed, (3) slow neutrons inducing fissions on fissile materials, (4) both scattered interrogation neutrons and newly-produced fission neutrons being collected by the detector array, (5) algorithms analyzing the pulse-train for discriminating between fission and interrogation neutrons, and finally (6) a qualitative and quantitative assay of fissile material being given.

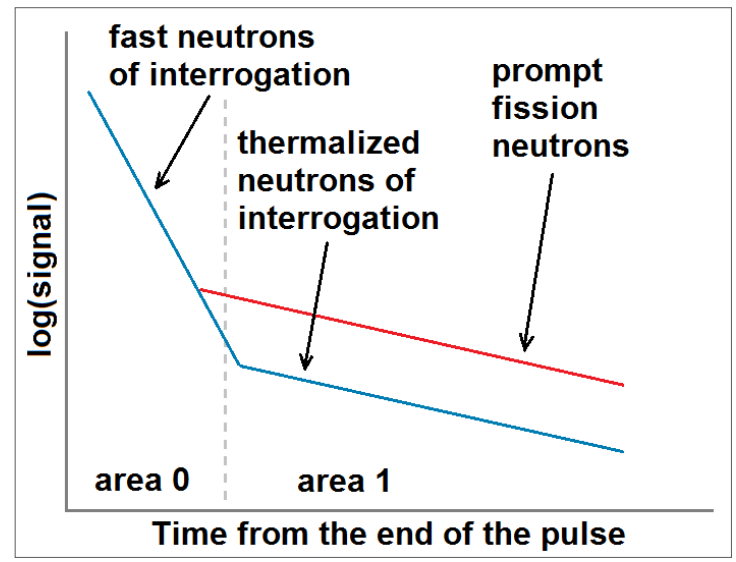

Figure 1. Theoretical Differential Die-Away Time Analysis (DDAA) signals against time in the presence of fissile material (red line) or other materials (blue line). The region of interest for detecting fissile material is labeled "area 1 " where differences between the curves can be observed.

The expected measured signal of a sample with and without fissile material is sketched in Figure 1. Such trends can be explained as follows: (i) fast interrogation neutrons are characterized by a certain decay time producing the area 0 (such behavior in time being independent by the presence 
of fissile material into the sample); (ii) the detection of fissile material is possible due to prompt neutrons emerging from induced thermal fission causing an increase of the signal (red curve) when fast interrogation neutrons disappear. If fissile material is not present, the expected signal is much lower (blue curve); (iii) the more the fissile mass, the more the distance between the two curves.

Based upon the different "die-away-time" of fission neutrons from interrogation neutrons, the DDAA is a very reliable technique in discovering fissile material hidden in packages, vehicles, or containers. The main issue is the implementation of the technique into a real-case scenario, i.e., number of devices needed (e.g., neutron generators, moderation systems, neutron detectors), their geometrical configuration, the minimum irradiation time for collecting a statistically significant signal, radiation protection requirements for the operators, and its possible remote handling for achieving the desired performances of the system. In particular, the main target for the designer of such kind of devices should be the Minimum Detectable Activity (MDA) of fissile material, i.e., the minimum quantity of fissile material for triggering the alarm as a nuclear threat. The NAI device is intended to overcome the limitations of similar systems [15-18] by (1) decreasing the MDA, (2) lowering the device in weight, and (3) getting real-time responses.

The NAI device is conceived as a modular instrument. In particular, it can be tailored by adopting different parts (i.e., neutron generator, detector arrays, moderator blocks) to get the best solution according to the particular measurement condition and sample to be analyzed. The availability of simulation tools like the Monte Carlo code for radiation transport MCNPX [19] allows to simulate the behavior of each part, permitting the user to optimize the experimental setup according to the required performances. For example, Figure 2 reports the sequential trials for the DDAA optimization for the measurement of small volume samples, namely the target goal of the NAI device into the EDEN Project. Each combination of parts to be analyzed (packages, cars, containers, and so on) needs special consideration, according - most of all - to the sample dimensions. Whatever the sample, simulations and measurements can be carried out and the optimal configuration identified.

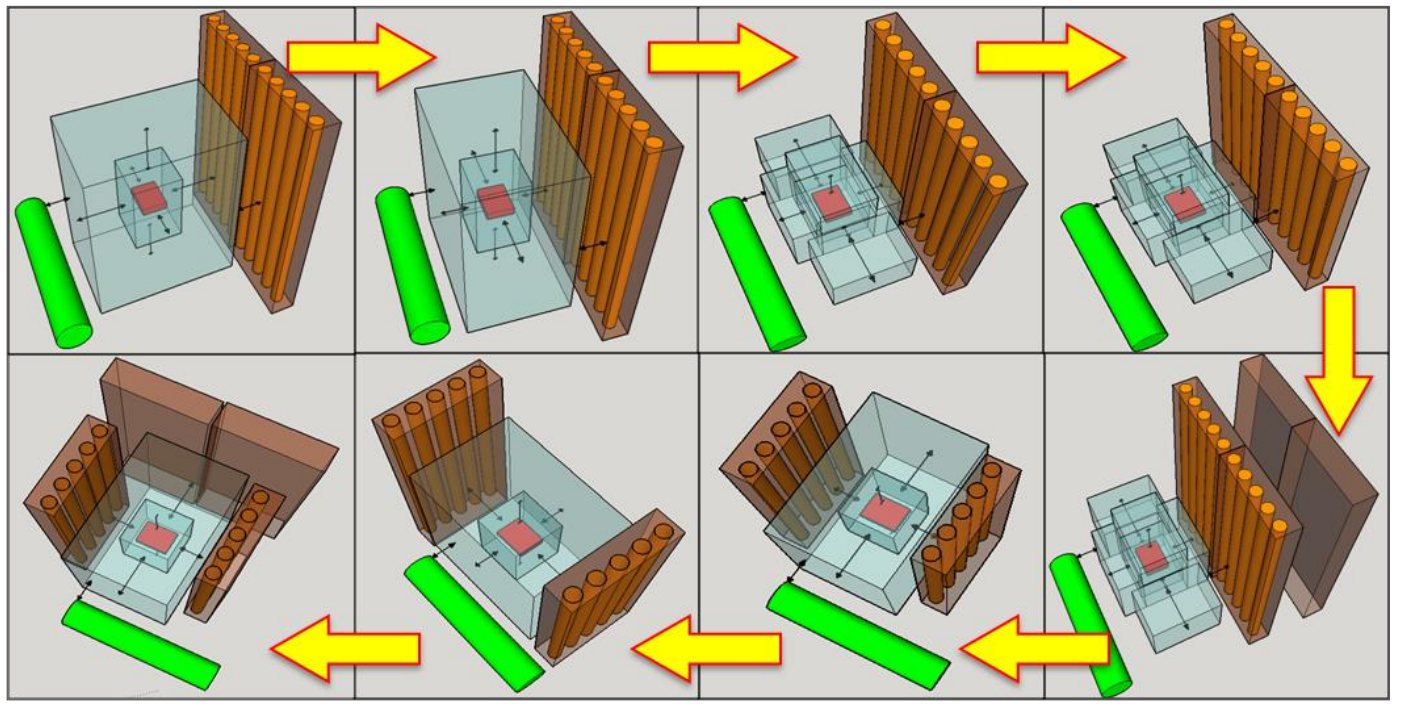

Figure 2. Example of sequential trials in the optimization process to get the best measurement configuration for small volume samples. In green the neutron generator, in transparent blue the moderating structure around the sample to be measured (red), in orange the ${ }^{3} \mathrm{He}$ tubes embedded into a polyethylene structure (in gray).

In the case of small volume samples, the starting point was an experimental setup for laboratory measurement proposed by Jordan et al. [15]. Such a geometric configuration has been reconstructed into MCNPX for validating the simulation code ability in reproducing the results reported by [15]. Such test has been an inspiration for identifying the best geometrical configuration for small volume 
samples measurement later on. In particular, starting from the original setup [15], we carried out an optimization process by means of Monte Carlo simulations (Figure 2) and each step of the process has been benchmarked by appropriate experimental measurements for assuring the ability of the simulations to reproduce the real-world case.

\section{Results and Discussion}

As previously stated, the goals of the design process were to get a device (1) light in weight, (2) characterized by MDA of the order of some grams of fissile material (3) detected in a measurement time so short that the device is able to provide answers in real-time. For comparison, the experimental setup by Jordan et al. [15] is able to detect $350 \mathrm{~g}$ of ${ }^{235} \mathrm{U}$ within a moderating structure of several hundreds of kilograms in mass.

The simulations suggest that decreasing the fissile mass to $2 \mathrm{~g}$ of ${ }^{235} \mathrm{U}$ implies a reduction of the system ability in discriminating the interrogation neutrons by the fission neutrons, the latter characterizing the fissile material. However, the difference between the interrogation signal and the fission signal (i.e., area 1 of Figure 1) would still be significant, opening the possibility for modifying the other parameters, such as geometry and moderators, to increase the system efficiency and the discrimination ability.

The reduction in mass of the moderating structures corresponds to a proportional decrease of the distance between the sample and the neutron source (i.e., the less the distance, the higher the neutron flux on the sample), between the sample and the detectors (i.e., the less the distance, the higher the detectors geometrical efficiency), maintaining an appropriate thickness of moderator (i.e., the slower the neutrons, the higher the probability of thermal fissions, on turn implying higher probability in detecting neutrons by means of the ${ }^{3} \mathrm{He}$ tubes). Therefore, such mass reduction optimizes the system efficiency and increases the discrimination ability between interrogation and fission neutrons.

Simulations and experimental trials provided evidence that a reduction up to about $30 \mathrm{~kg}$ of moderator still allows the neutron discrimination if cadmium sheets are added to enclose the detector system. In fact, cadmium absorbs most of thermalized interrogation neutrons and permits their discrimination from fission neutrons. In terms of energy, cadmium cuts neutrons with an energy of less than $0.5 \mathrm{eV}$, purging the polyethylene which detectors are enclosed in by such a contribution.

As shown in the first panel of Figure 2, in the original experimental setup the positions of the neutron generator, of the sample, and of the detectors are on the same axis, while the distance between the neutron generator and the detectors is the largest as possible. The simulations have revealed that a sample cavity is recommended instead. In fact, if the detectors surround the sample - as shown in the last panel of Figure 2 - the detection efficiency is the highest and this corresponds to a more efficient discrimination between interrogation and fission neutrons.

In the light of the previous considerations, the following experimental setup has been proposed:

- $\quad$ a neutron generator based on the d-t fusion reaction that produces neutrons of about $14 \mathrm{MeV}$;

- $10^{3} \mathrm{He}$ proportional counters with nominal pressure of 4 bar inserted inside two plates of high density polyethylene surrounded by a cadmium sheet and a layer of aluminum;

- two other plates of high density polyethylene surrounded by a layer of aluminum;

- a moderating structure composed by stacks of copier paper with a total mass of about $30 \mathrm{~kg}$.

The results of the simulation related to the final configuration reported in the last panel of Figure 2 are illustrated in Figure 3: the red curve corresponds to the case of a sample containing $392 \mathrm{~g}$ of depleted uranium with about $2 \mathrm{~g}$ of ${ }^{235} \mathrm{U}$, while the blue curve corresponds to a sample without SNMs. The analysis of the signal in the region between 500 and $1500 \mu$ s can be used to trigger an alarm, potentially uncovering the presence of fissile material in the sample. 


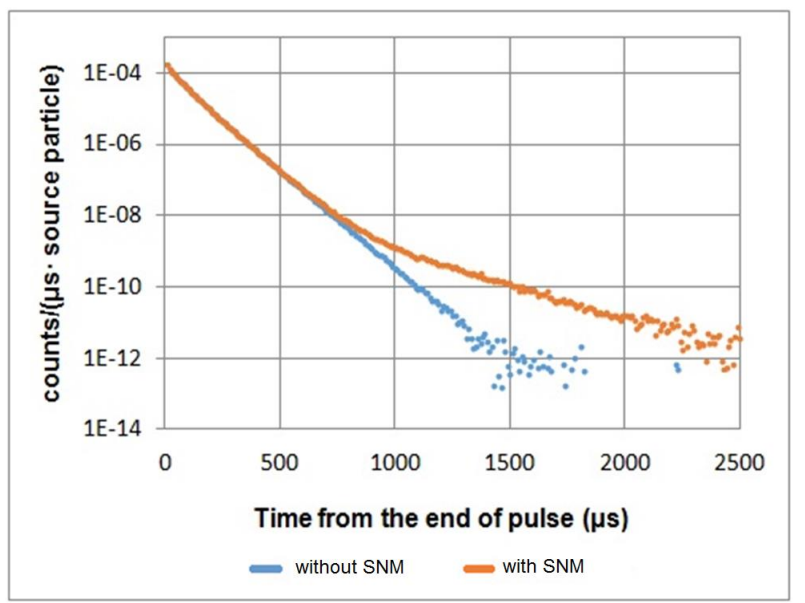

Figure 3. Simulated signals collected by the Neutron Active Interrogation (NAI) device of samples containing (red dots) and not containing (blue dots) Special Nuclear Materials fissile material.

The simulated results reported in Figure 3 have been confirmed experimentally in a live demo open to the public at the EDEN Project Demonstration RN4.1 and RN4.2 occurred on 29 and 30 September 2015 at ENEA Frascati Research Centre in Rome. Figure 4 shows the NAI device implemented on field. The experimental setup is made of several items (neutron generator and detectors blocks), each one is mounted on a trolley and each of them can be moved around the sample in the most appropriate configuration according to the specific measurement. A remote computer controls the execution of the measurements and the analysis software, in a safe position for the operator. The device is therefore adaptive: it is possible to embrace and measure different kind of samples with different sizes, as illustrated in Figure 5.

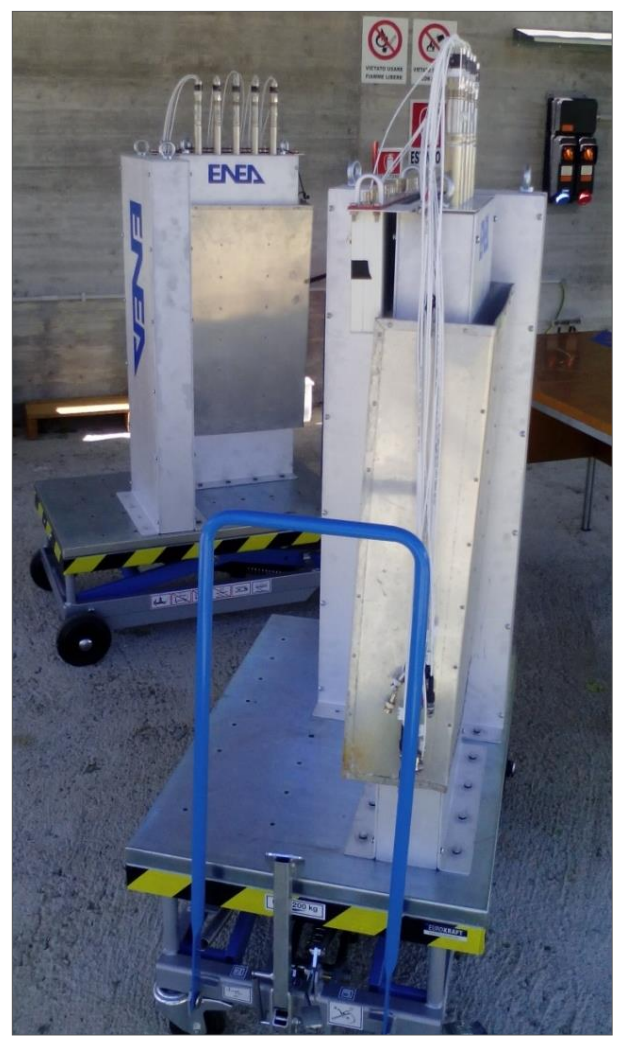

Figure 4. The NAI device experimental setup on the field. 


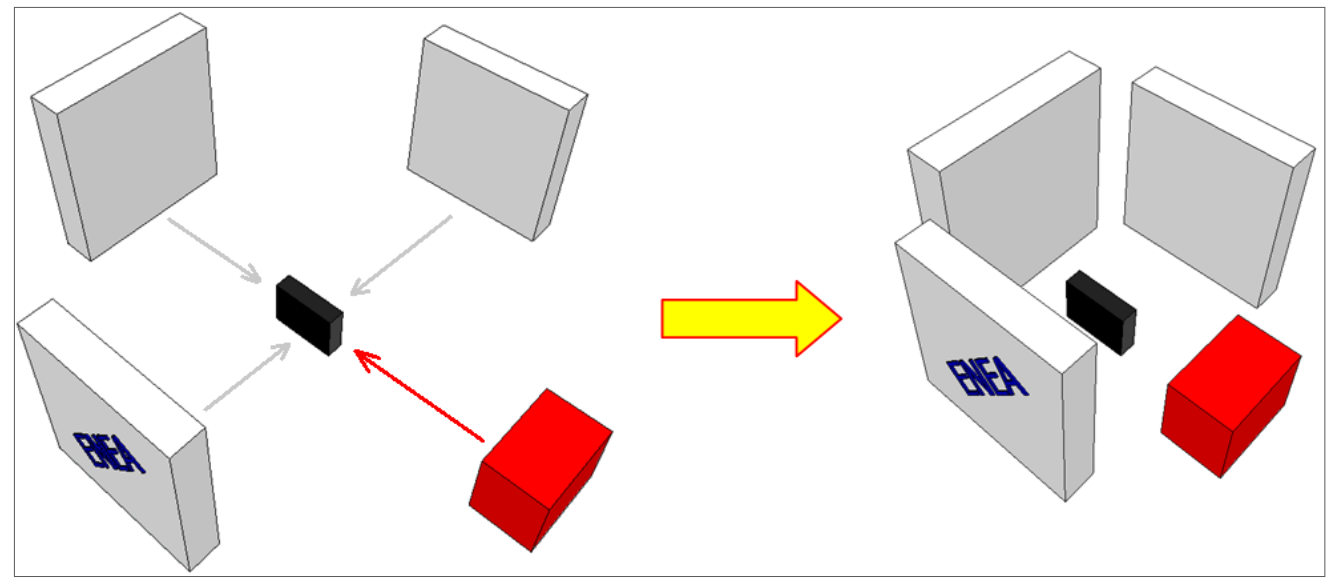

Figure 5. The NAI device adaptive principle: the suspected item (black box) is embraced by neutron generator (red box) and detectors blocks (gray boxes) each one mounted on a trolley or a remote operated vehicle. A computer remotely controls the measurement acquisition and the analysis software, providing a real-time response about the presence of a threat.

The experimental setup has been operated with the generator pulsing neutron at the minimum allowed frequency (i.e., namely $500 \mathrm{~Hz}$ ) with a $10 \%$ duty factor. It means that the generator triggers a neutron pulse every $2 \mathrm{~ms}$. The corresponding pulse is long one tenth of such time (i.e., $200 \mu \mathrm{s})$. Each neutron detector counts the neutrons in bins of $10 \mu \mathrm{s}$ by a custom fast electronic and a cumulative signal is built. A full measurement cycle takes between 1 to 2 seconds (e.g., if the interrogation time is $2 \mathrm{~s}$, the sample will be reached by 1000 bursts of neutrons, then 1000 pulses are detected). The cumulative signal (the sum over all detectors) is sent to a computer by means of an optical fiber connection. Here, the collected data are analyzed by a mathematical algorithm which compares the slope of the curve obtained from the sample measurement to a previously collected reference signal (afterwards referred as "background"), being the slope of descending part of the pulse related to the presence of fissile material.

During the reading of the pulse train, an appropriate control parameter discerns the irregular pulses to be rejected, while the remaining pulses are summed up to obtain a mean pulse that is representative of the measurement and characterized by a more significant counting statistics if compared to a single pulse of the train. The algorithm then compares the computed mean experimental pulse (from the sample) to the background (i.e., the empty cavity). If the former is significantly higher than the latter, the algorithm is able to discriminate if the fissile material is present in the sample. In particular, referring to measurement reported in Figure 6 and considering the $n=8$ bins at times $>560 \mu \mathrm{s}$, the difference between measurement and background pulses is calculated for each bin $\left(\Delta \mathrm{B}_{\mathrm{i}}\right.$ with $\mathrm{i}=1, \ldots, \mathrm{n})$. If $\Delta \mathrm{B}$ is the average of the $\Delta \mathrm{B}_{\mathrm{i}}$ and $\sigma_{B}$ the corresponding standard deviation of the mean, a detection of fissile material is considered positive if $\Delta B / \sigma_{B}>3$.

Figure 6, left, shows the signal measured when a sample containing fissile material is analyzed (in this case $6 \mathrm{~g}$ of ${ }^{235} \mathrm{U}$ ). With reference to the simulated results reported in Figure 3, in Figure 6 a deflection of the measured signal from the background (measurement at empty cavity) shows up, with $\Delta \mathrm{B} / \sigma_{\mathrm{B}}=4.3$. The slight difference currently observed in these two curves is due to the temporary geometry of the cadmium sheets adopted in the field operations that do not completely enclose the detector system preventing to get the performances expected and shown in the simulations illustrated in Figure 3. Figure 6, right, shows a similar comparison when a sample not containing fissile material is analyzed: as can be seen, the measured signal does not deflect from the background. 


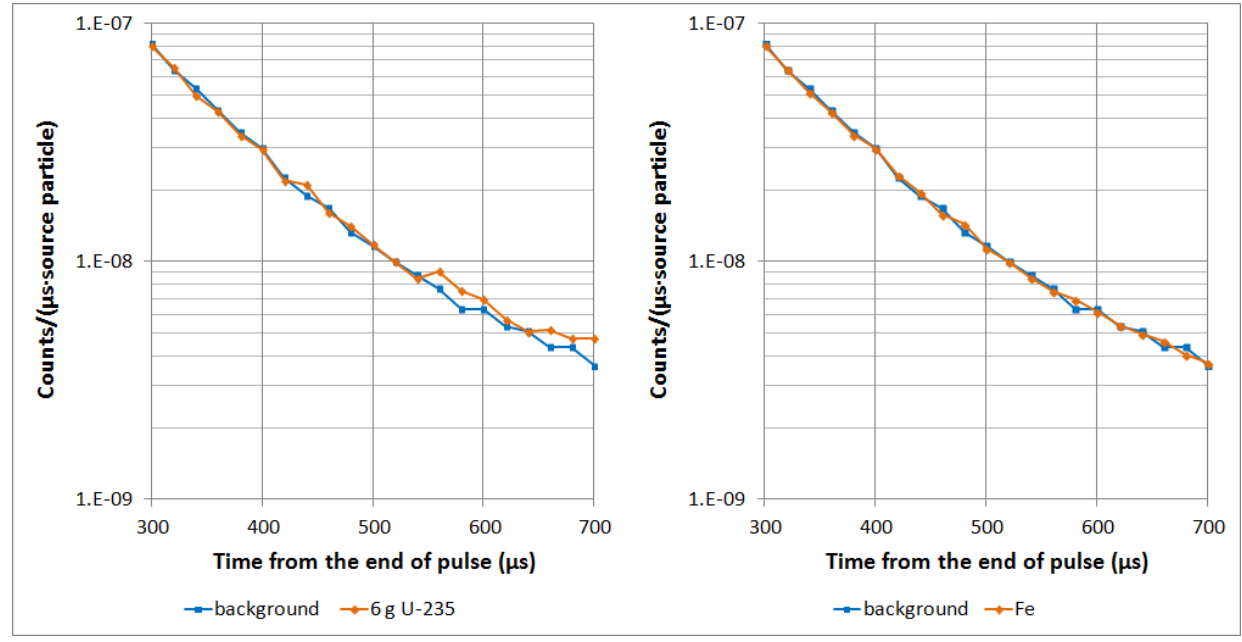

Figure 6. Experimental results of a measurement performed by the NAI device. The left panel shows the deflection of the measured signal coming from a sample with fissile material compared to background signal (when cavity is empty). The right one shows a similar comparison where the measured sample does not contain fissile material. The relative error $\sigma_{B} / \Delta B=0.09$ for each of the three train pulses illustrated.

\section{Conclusions}

The ENEA NAI device is a tool designed to improve CBRNE resilience capacity of EU society. In particular, NAI is built to uncover radioactive and nuclear threats also in the form of IEDs, the so-called "dirty bombs". The current development stage of the NAI device provides a positive detection of fissile material in the case of a sample containing about $6 \mathrm{~g}$ of ${ }^{235} \mathrm{U}$ hidden in a package through a transportable system, light in weight, with a real-time response. It is a prototype ready for an industrial integrated system (e.g., such as a luggage inspection system) and a promising base for further developments.

The experimental setup illustrated here is currently undergoing new developments for reducing the MDA by means of a better geometry of the cadmium sheets enclosing the neutron detectors and, at the same time, reducing the instrument mass and dimensions. Moreover, given the expertise of all the ENEA personnel involved in the project, future developments also include the extension of the capability of the NAI device by implementing the simultaneous detection of explosive materials.

Acknowledgments: The computing resources and the related technical support used for this work have been provided by CRESCO/ENEAGRID High Performance Computing infrastructure and its staff [20]. CRESCO/ENEAGRID High Performance Computing infrastructure is funded by ENEA, the Italian National Agency for New Technologies, Energy and Sustainable Economic Development and by Italian and European research programmes, see http:/ / www.cresco.enea.it/english for information. The authors warmly thank the ENEA Casaccia Research Center TRIGA RC1 personnel for their support and cooperation during the experiments. The authors thank SOGIN S.P.A. in particular Ipu Plant (Plutonium Plant), personnel for their cooperation to provide ${ }^{3} \mathrm{He}$ neutron detector.

Author Contributions: All authors equally contributed to the presented work and the paper preparation.

Conflicts of Interest: The authors declare no conflict of interest.

\section{References}

1. Datema, C.P.; Bom, V.R.; van Eijk, C.W.E. Landmine detection with the neutron backscattering method. IEEE Trans. Nucl. Sci. 2001. [CrossRef]

2. Buffler, A.; Brooks, F.D.; Allie, M.S.; Bharuth-Ram, K.; Nchodu, M.R. Material classification by fast neutron scattering. Nucl. Instrum. Methods Phys. Res. Sect. B 2001, 173, 483-502. [CrossRef] 
3. Lee, W.C.; Mahood, D.B.; Ryge, P.; Shea, P.; Gozani, T. Thermal neutron analysis (TNA) explosive detection based on electronic neutron generators. Nucl. Instrum. Methods Phys. Res. Sect. B 1995, 99, 739-742. [CrossRef]

4. Buffler, A. Contraband detection with fast neutrons. Radiat. Phys. Chem. 2004, 71, 853-861. [CrossRef]

5. Bystritsky, V.M.; Ivanov, A.I.; Kadyshevsky, V.G.; Kobzev, A.P.; Nikitin, V.A.; Rogov, Y.N.; Sapozhnikov, M.G.; Slepnev, V.M.; Sissakian, A.N.; Vlasov, N.V. Study of the Associated Particle Imaging technique for the hidden explosives identification. In Proceedings of the International Conference on Requirements and Technologies for the Detection, Removal and Neutralization of Landmines and UXO, EUDEM2-SCOT-2003, Brussels, Belgium, 15-18 September 2003.

6. Runkle, R.C.; White, T.A.; Miller, E.A.; Caggiano, J.A.; Collins, B.A. Photon and neutron interrogation techniques for chemical explosives detection in air cargo: A critical review. Nucl. Instrum. Methods Phys. Res. A 2009, 603, 510-528. [CrossRef]

7. Hakonen, A.; Andersson, P.O.; Schmidt, M.S.; Rindzevicius, T.; Kall, M. Explosive and chemical threat detection by suface-enhanced Raman scattering: A review. Anal. Chim. Acta 2015, 893, 1-13. [CrossRef] [PubMed]

8. Crespy, C.; Duvauchelle, P.; Kaftandjian, V.; Soulez, F.; Ponard, P. Energy dispersive X-ray diffraction to identify explosive substances: Spectra analysis procedure optimization. Nucl. Instrum. Methods Phys. Res. A 2010, 623, 1050-1060. [CrossRef]

9. SODERN. Unattended Luggage Inspection System (ULIS). Available online: http://www.sodern.com/sites / en/ref/ULIS_80.html (accessed on 6 June 2016).

10. SODERN. Neutron Induced Prompt Photometer System (NIPPS). Available online: http:/ / www.sodern. com/sites/en/ref/NIPPS_82.html (accessed on 6 June 2016).

11. Clifford, E.T.H.; McFee, J.E.; Ing, H.; Andrews, H.R.; Tennant, D.; Harper, E.; Faust, A.A. A militarily fielded thermal neutron activation sensor for landmine detection. Nucl. Instrum. Methods Phys. Res. A 2007, 579, 418-425. [CrossRef]

12. Airbus Defence \& Airspace. Sonex-P; Airbus Defence \& Airspace. Available online: http://northamerica. airbus-group.com/north-america/usa/Airbus-Defense-and-Space/SONEX-P/Overview.html (accessed on 6 June 2016).

13. Caldwell, J.T.; Kunz, W.E.; Atencio, J.D. Apparatus and Method for Quantitative Assay of Generic Transuranic Wastes from Nuclear Reactors. U.S. Patent 4,483,816, 20 November 1984.

14. Ensslin, N. Principles of Neutron Coincidence Counting. In Passive Non-Destructive Assay of Nuclear Materials; NUREG/CR-5550; United States Nuclear Regulatory Commission: Washington, DC, USA, 1991.

15. Jordan, K.A.; Gozani, T.; Vujic, J. Differential die-away analysis system response modeling and detector design. Nucl. Instrum. Methods Phys. Res. A 2008, 589, 436-444. [CrossRef]

16. Jordan, K.A.; Vujic, J.; Gozani, T. Remote thermal neutron die-away measurements to improve Differential Die-Away Analysis. Nucl. Instrum. Methods Phys. Res. A 2007, 579, 407-409. [CrossRef]

17. Jordan, K.A.; Gozani, T. Detection of ${ }^{235} \mathrm{U}$ in hydrogenous cargo with Differential Die-Away Analysis and optimized neutron detectors. Nucl. Instrum. Methods Phys. Res. A 2007, 579, 388-390. [CrossRef]

18. Jordan, K.A.; Gozani, T. Pulsed neutron differential die away analysis for detection of nuclear materials. Nucl. Instrum. Methods Phys. Res. B 2007, 261, 365-368. [CrossRef]

19. MCNP-A General Purpose Monte Carlo Code for Neutron and Photon Transport; LA-12625-M; Briesmeister, J.F., Ed.; Los Alamos National Laboratory: Los Alamos, NM, USA, 1993.

20. Ponti, G.; Palombi, F.; Abate, D.; Ambrosino, F.; Aprea, G.; Bastianelli, T.; Beone, F.; Bertini, R.; Bracco, G.; Caporicci, M.; et al. The role of medium size facilities in the HPC ecosystem: The case of the new CRESCO4 cluster integrated in the ENEAGRID infrastructure. In Proceedings of the 2014 International Conference on High Performance Computing and Simulation, HPCS 2014, Bologna, Italy, 21-25 July 2014; pp. 1030-1033.

(C) 2016 by the authors; licensee MDPI, Basel, Switzerland. This article is an open access article distributed under the terms and conditions of the Creative Commons Attribution (CC-BY) license (http://creativecommons.org/licenses/by/4.0/). 